\title{
Les aspects ethnographiques et patrimoniaux du chanvre dans la Sarthe
}

\author{
Serge BERTIN \\ Docteur en ethnologie, chercheur associé à l'UMR TEMOS, Le Mans-Université
}

Durant un siècle, le chanvre illustra l'une des activités économiques les plus prospères et les plus valorisantes du département de la Sarthe. Pourtant, son histoire reste globalement méconnue, même si des chercheurs ont pu, parfois de façon succincte, en aborder certains aspects dans le cadre de leurs propres champs d'études. L'érudit André Bouton, auteur d'un monumental ouvrage, Le Maine Histoire économique et sociale, Le Mans, impr. Monnoyer, 1974, lui consacra seulement l'équivalent de deux ou trois pages au fil de ses cinq volumes (de la Préhistoire au $\mathrm{XIX}$ siècle). Dans sa thèse de doctorat Louis Simon étaminier (1741-1820) dans son village du Haut-Maine au Siècle des Lumières, 1982, LXXXVIII-655 pages, Anne Fillon le cita à plusieurs reprises lorsqu'elle analysa les inventaires qu'elle avait puisés dans les minutes notariales. François Dornic, auteur d'une thèse de doctorat L'industrie textile dans le Maine et ses débouchés internationaux (1650-1815), Pierre Belon, 1955, XXVI-316 pages, consacra d'importants chapitres à la fabrication et la commercialisation de ses toiles. Enfin, Jeanne Dufour, dans sa thèse de doctorat Agriculture et agriculteurs dans les campagnes mancelles Le devenir des régions agricoles, 1981, 596 pages, traita largement de sa culture, dans une approche à la fois géographique et chronologique. Quant aux sources archivistiques, malheureusement éparses, les deux plus importantes se trouvent dans la sous-série $7 \mathrm{M}$ (Agriculture) des Archives départementales de la Sarthe pour l'une et au sein de la Société Agriculture Sciences et Arts de la Sarthe pour l'autre, sous la forme de mémoires, de lettres et registres de délibérations.

Enfin, l'autre pan de la connaissance de cette plante réside dans les mémoires, mémoire des anciens chanvriers, mais aussi mémoire des lieux où ils la travaillèrent.

Histoire, mémoires et paysages constitueront les trois axes autour desquels s'articulera cette communication.

\section{L'histoire du chanvre en Sarthe}

Longtemps, tout au moins jusqu'au début du XIX ${ }^{\mathrm{e}}$ siècle, le Haut-Maine qui, en 1790 , donnera la majeure partie du département de la Sarthe, occupa une place modeste dans la production du chanvre en France. Dans une pièce de terre bien fumée, qu'ici on appelait un cloteau, on cultivait un chanvre essentiellement destiné à la consommation locale : cordages, ficelle, toiles pour l'habillement ou pour la confection des draps, etc.

C'est au XIX $\mathrm{X}^{\mathrm{e}}$ siècle que, pour la Sarthe, se produisit le miracle. Pour l'ensemble de la France, le chanvre entame son déclin à partir de 1840, les surfaces ensemencées passant de 176000 hectares à cette date, à 100000 environ une vingtaine d'années plus tard. Et la dégringolade ne cesse de s'accélérer. Dans le même temps, le département de la Sarthe, qui avait connu un très fort accroissement aux lendemains de la Révolution française, passant de 3000 hectares en 1811 à 8000 en 1837, poursuit sa progression jusqu'en 1866. En d'autres termes, la Sarthe se consacre activement à la culture du chanvre et à sa transformation pendant que le 
reste du pays l'abandonne. Alors qu'il représentait moins de $2 \%$ de la production nationale au début du XIX siècle, il dépasse $10 \%, 50$ ans plus tard. Et la tendance s'accentue. Même lorsque, à la fin du $\mathrm{XIX}^{\mathrm{e}}$ siècle, le chanvre sarthois amorce lui aussi, son déclin, très lent, la situation française s'est tellement dégradée qu'il fait encore figure d'exception. Durant la Première Guerre mondiale, la Sarthe est le premier producteur national. Elle le restera jusqu'aux années 1960, assurant, certaines années, la quasi-totalité de cette culture, seulement concurrencée par le Maine-et-Loire et I'Indre-et-Loire voisins. L'euphorie règne donc dans le monde chanvrier sarthois. Pourtant, dès l'année 1961, un article de Jeanne Dufour ${ }^{1}$, fait l'effet d'une douche froide. Au début de la décennie suivante, la mort annoncée par la géographe semble sur le point de se réaliser: la plante n'intéresse plus que 33 agriculteurs sarthois qui, au total, ne lui accordent que 83 hectares. Certains, faute d'avoir pu écouler leur récolte en ce temps-là, la conservent encore, de nos jours, dans le vain espoir de trouver preneur.

On comprend que, dans ces conditions, le souvenir reste vif dans les mémoires de nombre de chanvriers sarthois pour lesquels le chanvre (ils disent le " chanbre ») était sans conteste "le bonheur du paysan ". C'est une chance pour nous autres, chercheurs.

\section{Mémoires vivantes du chanvre}

Trois principales campagnes de collecte des témoignages de celles et de ceux qui cultivèrent le chanvre ont été réalisées ; elles se déroulèrent en 1986, en 20012002 et 2011. Près d'une trentaine de personnes furent interrogées et leurs propos enregistrés sur quatorze cassettes audio qui représentent un total d'une vingtaine d'heures.

Leurs souvenirs se révèlent particulièrement précis et fiables, même s'ils apparaissent souvent empreints de nostalgie. Grâce à eux, soumis aux règles habituelles de l'analyse critique, nous pouvons reconstituer les étapes de la culture du chanvre. Sous nos yeux, se déroule le film de pratiques aujourd'hui ignorées.

Ainsi, parlent-ils, avec amour, de la manière dont ils préparaient leur pièce de terre. "Fallait que l'guéret, y saye comme de la cendre, et pis, on attendait que la terre, elle fleurisse ${ }^{2} », \mathrm{Et}$, alors seulement fin avril, quand tout risque de gelée semblait écarté ${ }^{3}$, ils semaient le « chènevis ». Un usage consistait à planter une branche de buis bénit des Rameaux, à l'entrée du champ que l'on venait d'ensemencer pour le préserver des pluies d'orages, fréquentes au mois de mai.

Quatre mois plus tard, vers la Saint-Louis, arrivaient les hordes de tâcherons venus de la ville, ou bien d'ailleurs qui, du petit matin jusqu'à la nuit tombante, se livraient au labeur harassant de l'arrachage. Mis en poignées, les pieds, hauts de trois mètres ou plus, étaient ficelés à la base et à la tête, à l'aide de liens faits de petits brins de chanvre. Les mains en sang, le côté du corps irrité par le frottement de la plante, l'esprit égaré par la troublante odeur du végétal, les arracheurs allaient jusqu'à faire mille poignées dans leur journée. Les plus anciens racontent qu'autrefois, il fallait arracher d'abord les pieds mâles, qu'ici on appelait les pieds

\footnotetext{
1 DUFOUR Jeanne, "Une culture moribonde, le chanvre français », L'information géographique, $\mathrm{n}^{\circ} 4$, Paris, Armand Colin, 1961, p. 139-154.

2 BLIN G., Laigné-en-Belin, entretien enregistré, année 2011.

${ }^{3}$ Tout risque aura véritablement disparu après les « saints de glace », les 11, 12 et 13 mai.
} 
femelles, en raison de leur plus petite taille, et une semaine ou deux plus tard, les pieds femelles, porteurs de graines, plus puissants et pour cette raison appelés pieds mâles. À partir des années 1950, l'apparition des variétés monoïques ${ }^{4}$ aux fleurs mâles et femelles disposées sur le même pied, les chanvres "boubiques ", simplifieront grandement le travail.

Dans la foulée, les chanvres arrachés étaient immergés pour subir le rouissage. Celui-ci s'effectuait soit dans une mare creusée à cet effet, qu'on appelait rouisson ou routoir, soit, de préférence, dans l'eau courante d'une rivière. II fallait d'abord « faire le Bon Dieu », c'est-à-dire confectionner une croix, ou bien un carré, à l'aide de poignées de chanvre, puis jeter le «Bon Dieu » à l'eau en prenant soin de le relier à la terre ferme par un solide cordage. On "chargeait le Bon Dieu » en disposant dessus les gerbes de chanvre de manière à former une sorte de radeau qu'on appelait, selon les lieux, une " tuilée », une " douétée » ou bien une " culée ». Cette barge de chanvre, dont la forme s'apparentait à celle d'une pyramide tronquée renversée devait, pour rester dans l'eau, être lourdement lestée. II fallait, pour cela, la charger de grosses pierres, portées à la main. Huit à neuf tonnes étaient nécessaires par « tuilée ». Au bout de huit jours environ, le rouissage était terminé, c'est à dire que la décomposition de la partie ligneuse et, accessoirement celle des feuilles était achevée, la " tuilée " était démontée : les pierres retirées une à une, les poignées de chanvre évacuées pour être séchées. Cette opération se pratiquait sur les chaumes ou sicots des blés fraîchement coupés. De l'arrière du " baniau », une équipe tirait les poignées gluantes et malodorantes et les déposait, à intervalles réguliers, sur le sol. Femmes et enfants coupaient les liens et égaillaient les pieds en lignes.

« Nous, quéniaux, c'était notre boulot. Avec notre couteau, toc... toc... La poignée, ça prenait 80 centimètres. Et puis, on laissait 20 centimètres entre chaque poignée... parce qu'après, il fallait tourner avec un broc à deux doigts. Ça séchait vite, hein ! Au bout de deux jours, on retournait. Souvent, quand on le retournait, le lendemain, on le ramassait. Oui, s'il était sec ! J'ai vu, des fois, le retourner le soir, au clair de lune... tard... c'était beau. Le lendemain... ramassé ${ }^{5} ! »$.

\section{Ramassé pour l'hiver dans les hangars}

C'est en janvier que les chanvriers sarthois effectuaient la phase finale de la transformation du chanvre. Les gerbes étaient disposées dans des fours. Elles y passaient la nuit à subir une dessiccation qui évacuait toute l'eau et fragilisait les restes de parties ligneuses non détruites par le rouissage. Au petit matin, brûlantes, elles passaient entre les mains des "brayeurs ", des ouvriers agricoles spécialisés dans ce type d'opération. Chaque pied était glissé entre les rouleaux dentelés de la " brayeuse », engagé par " l'affoureux » et retiré par le " tire au cul ». Il était ensuite disposé entre les mâchoires de la "braye " pour achever d'évacuer les derniers éclats d'écorce qu'on appelle ici les "grettes». Ne restait plus, à la main du " brayeur » que la filasse qu'il nouait joliment pour en faire un « tord ».

Convoqués par les marchands à la gare la plus proche, les chanvriers livraient leur cargaison de chanvre.

\footnotetext{
${ }^{4}$ Une plante monoïque possède des fleurs mâles et femelles en des endroits différents d'un même pied.

${ }^{5}$ RousSEAU Y., Souillé, entretien enregistré, année 2011.
} 
«C'était auquel faisait les plus beaux paquets pour vendre... mais aussi pour la gloire. On chargeait la veille. Quand les paquets étaient tous bien ficelés, dès qu'on passait un peu la hauteur, on retapait tous les rangs avec une pelle-bêche bien propre, pour tout bien tasser droit. C'était un mur... un mur dessiné, qu'on amenait ${ }^{6}$ ! .

Les retours étaient souvent bien arrosés. Georges Blin, de Laigné-en-Belin, se souvient d'une mésaventure alors qu'un jour, au café, il fêtait trop dignement sa livraison de chanvre :

«Et ma jument, pourtant, elle n'est pas facile. Eh bien, ils se sont permis de la dételer et ils l'ont mise à l'envers, la tête à la place du derrière! ${ }^{7}$ ".

Souvenirs, mais pas seulement. Des traces encore bien concrètes existent de cette épopée chanvrière.

\section{Les fours à chanvre}

Depuis le début des années 1980, et jusqu'à aujourd'hui, des campagnes de photographie des fours à chanvre en Sarthe ont été menées. Plusieurs centaines de clichés sont actuellement conservées au domicile de l'auteur de cette communication. S'y trouvent aussi les fiches signalétiques des quelque cinq cent fours qui ont été recensés à la fin des années 1990. Cette opération, lancée à la demande de l'association de Sauvegarde des Fours à Chanvre, présidée par Roger Malassigné, fut assurée par les étudiantes et étudiants du DUFCEP ${ }^{8}$ sous la conduite d'Anne Fillon, alors directrice du CUEP $^{9}$ du Mans. Ces données, abondantes et précises, ont permis d'élaborer un tableau du parc immobilier chanvrier en cette fin de $X X^{e}$ siècle. En voici les grandes lignes.

Les fours à chanvre se répartissent entre deux grandes catégories, les carrés et les ronds, mais tous présentent la même disposition : une chambre de chauffe à l'intérieur de laquelle se trouve une corbeille à coke, et une chambre de séchage séparée de la précédente par un plancher à claire-voie. Illustration saisissante de la capacité d'adaptation au milieu, propre au monde traditionnel rural : dans les fours construits sur talus, la salle de séchage, qui contient les gerbes, donne sur le terrain où est installée la loge, alors que le foyer débouche sur le chemin, en contrebas.

Les fours ronds, avec $66 \%$ de l'effectif total, sont les plus répandus. Ils se présentent sous la forme de tourelles, les deux portes décalées définissant avec certitude leur fonction. Les uns trapus, les autres élancés, ils affichent des dimensions très contrastées dont les diamètres extrêmes varient de 2,04 à 4,35 mètres. Les couvertures se déclinent selon toutes les nuances possibles. Ce peut être un dôme de maçonnerie, ou une simple dalle de béton. Généralement plat, le sommet des fours les plus anciens et des plus rustiques était composé d'une masse de terre ou bien de sable ${ }^{10}$, recouverte d'une épaisse couche végétale faite de vieux foin, de paille et surtout d'herbe :

\footnotetext{
${ }^{6}$ BRAULT A., Beaumont-sur-Sarthe, entretien enregistré, année 1986.

${ }^{7}$ BLIN G., Laigné-en-Belin, entretien enregistré, année 2011.

${ }^{8}$ Diplôme Universitaire de Formation à la Connaissance et à l'Exploitation du Patrimoine $(\mathrm{Bac}+3)$, créé au Mans en 1990.

${ }^{9}$ Centre Universitaire d'Éducation Permanente, Le Mans-Université.

${ }^{10}$ FALAIS L., Le Mans, entretien noté sur fiche, année 1986. Au-dessus du plafond de son four, il y avait trois mètres cubes de sable, le tout recouvert de paille.
} 
« de la guinche ${ }^{11}[\ldots]$ oui, de la guinche... de la grande herbe, toute longue. [...] De la plante humide [...] on mettait ça tous les ans sur le fourniau. [...] II y avait un coin marécageux. Bin, tous les ans, on le fauchait à la faux et on le ramassait pour le mettre. II fallait le recharger, parce que ça foulait. Et puis ça pourrissait quand même un petit peu. Alors, on le rechargeait tous les ans pour que ça s'égoutte. Et puis ça se foulait. Oui, tous les ans ${ }^{12} »$.

D'autres fours portent une toiture à deux pentes, parfois quatre, faites des matériaux les plus divers: tuiles, ardoise, fibrociment et, bien souvent, plaques de tôles ondulées. Mais le type le plus fréquemment rencontré, c'est le modèle conique, parfois à six ou huit pans coupés, éventuellement surmonté d'un épi de faîtage en zinc, couvert de petites tuiles plates ou, dans une moindre mesure, d'ardoises.

Sur une base globalement circulaire, ou plus précisément ovale, un four offre une disposition tout à fait originale. Situé au Pont, à Lucé-sous-Ballon, il comporte, accolées, deux salles de chauffe et de séchage. Toujours dans le registre des curiosités, signalons, au lieu de la Borde, à Bernay-en-Champagne, l'existence d'un four à trois niveaux : une chambre de chauffe à la base et deux, superposées, pour le séchage.

Les fours carrés, moins nombreux, n'en représentent pas moins le tiers des édifices recensés en Sarthe. On en trouve partout dans les zones chanvrières et, eux aussi, présentent, dans leurs dimensions, de fortes disparités ; les plus vastes, avec 4 mètres de côté, font exactement le double des plus petits. Leur couverture se réduit généralement à une seule pente, parfois deux, en maçonnerie, en ardoises ou, là aussi, en tôle ondulée. Les quelques fours carrés doubles que compte le département, ne se rencontrent que dans le Belinois, pour des raisons spécifiques qui tiennent aux structures d'exploitation propres à cette région. Rien, au premier coup d'œil, ne permet de déceler la fonction chanvrière de toutes ces banales bâtisses reconverties, aujourd'hui, en abris ou remises à matériels.

Le mortier jaune, fait de sable mêlé à la chaux, qui donne cette belle uniformité aux bâtiments ruraux de la région a le plus souvent disparu. Qu'ils en aient été recouverts, ou pas, presque tous ont été construits avec des produits tirés du soussol local : grès "roussard " au nord-ouest, calcaire dans la Champagne mancelle, rognons de silex dans une grande partie du département; quelques uns seulement sont faits de briquettes industrielles. Un bloc de grès ici, des briques pleines disposées verticalement ailleurs, une solide pièce de bois à la rigueur, marquent, en guise de linteau, l'encadrement supérieur de l'ouverture. Quant aux portes, elles sont faites d'un métal épais, susceptible de résister à la chaleur intérieure.

Quelques fours particulièrement remarquables survivent en Sarthe. Ce sont les fours en torchis qu'on appelle ici " en terrasse ", c'est à dire faits " d'argile montée avec de la bruyère ${ }^{13}$. Sur un soubassement de pierres délimitant le foyer, des poteaux de bois sont disposés tous les 30 centimètres environ, reliés entre eux par un entrelacs de baguettes de châtaignier, le tout noyé dans le torchis. II y en avait encore une bonne douzaine, dans le département, dans les années 1990. En 2012, on peut encore en voir au Buisson sur la commune d'Aigné, au Coq gris à Congédes-Guérets, à la Huronnerie à Saint-Marceau, à La Chapuisière à Souillé. Les autres ont disparu, abandonnés aux intempéries ou bien, plus brutalement, détruits par l'ignorance de leur propriétaire.

\footnotetext{
11 Sorte de carex.

12 Rousseau Y., Souillé, entretien enregistré, année 2011.

${ }^{13}$ FALLAIS L., Le Mans, année 1986.
} 
En 2001-2002, des étudiantes et étudiants du DESS « Valorisation du patrimoine et développement territorial " réalisèrent, dans le cadre de leur formation, une mission visant à étudier les perspectives de prise en compte de ce patrimoine exceptionnel que représentent les fours à chanvre. Les résultats de leurs travaux, porteurs d'espoir, ne purent malheureusement aboutir car l'association Sauvegarde des Fours à Chanvre, susceptible de porter un projet, entra bientôt en sommeil, décimée par la disparition de ses animateurs les plus actifs.

En ce début de $X X I^{e}$ siècle, le chanvre, qui a fait la richesse des Sarthois a cessé d'en être l'emblème, même si le siège du Syndicat des Producteurs de Chanvre reste toujours domicilié au Mans. Aujourd'hui il ne s'arrache plus, il ne se rouit plus et il ne se broie plus. Certes, on peut encore le trouver sur quelques parcelles, ici ou là... mais il n'est plus tout à fait le même. Semé, coupé puis expédié, comme n'importe quelle autre plante, il a perdu toute sa spécificité.

Aussi, nous vous en prions, lorsque vous traverserez le département, si vous trouvez sur votre route un de ces vieux fours à chanvre dressé comme un totem, arrêtez-vous. Peut-être vous racontera-t-il son histoire et celle de la belle plante qui fit la fierté des gens d'ici.

\section{Annexes}

Figure 1 - L'arrachage du chanvre. Début du $X X^{e}$ siècle. Carte postale, collection personnelle de l'auteur

Figure 2 - Le rouissage du chanvre sur l'Orne Saosnoise. Début du XX $x^{\mathrm{e}}$ siècle. Carte postale, collection personnelle de l'auteur

Figure 3 - Le broyage du chanvre dans la loge. Début du $X X^{e}$ siècle. Carte postale, collection Marie-Paule Gesland

Figure 4 - Fiche de recensement d'un four : le Buisson à Aigné (recto), 1997. Cliché Dorizon et Dubray. Collection personnelle de l'auteur

Figure 5 - Carte de localisation des fours à chanvre dans la Sarthe (hors Belinois). Collection de l'Association Sauvegarde des fours à chanvre

Figure 6 - Four à chanvre à couverture végétale, Neuville-sur-Sarthe. Croquis de Paul Cordonnier Détrie, années 1930-40. Collection personnelle de l'auteur

Figure 7 - Un four à chanvre au nord du Mans, 2011. Cliché Serge Bertin

Figure 8 - Four double de Couleuvrault, Téloché (Belinois), 2011. Cliché Serge Bertin

Figure 9 - Four double du Pont après restauration, Lucé-sous-Ballon, 2009. Cliché Serge Bertin

Figure 10- Four du Buisson après restauration, Aigné, 2019. Cliché J. Cl. Paumier 
